\title{
Experimental Research on Multi-Wavelength FBG Fabrication Based on Multiple Exposure
}

\author{
Jingsheng $\mathrm{LV}^{1^{*}}$, Xiaolei ZHANG ${ }^{1}$, Haifeng $\mathrm{QI}^{1}$, Jian Guo ${ }^{1}$, \\ Gangding $\mathrm{PENG}^{1,2}$, and Chang WANG ${ }^{1}$
}

\author{
${ }^{1}$ Key lab of optical fiber sensing technology of Shandong, Jinan, 250001, China \\ ${ }^{2}$ School of Electrical engineering and telecommunications, University of New South Wales, Sydney, Australia 2052 \\ *Corresponding author: Jingsheng LV_Ｅ-mail: 13869101310@163.com
}

\begin{abstract}
A fabrication method of the multi-wavelength fiber grating (FBG) was introduced. Using the scan exposure method, the multi-wavelength FBG can be successfully manufactured through applying different tensile forces during the multiple exposures process on the same fiber. Experiment results show that the position and the overlap of different sub FBGs will greatly affect the spectrum of every sub FBG. The spectrum of each sub FBG will be affected by short wave oscillation unless the lengths and positions of all sub FBGs are fully overlapped. For hydrogen loaded fiber, the wavelength and reflectivity of the $n$th level FBG will increase as the $(n+1)$ th level FBG is written. But for germanium doped photosensitive fiber, multiple exposure will increase the wavelength of previous sub FBGs while decrease the reflectivity of all sub FBGs. Through well distributing exposure intensity of every sub FBGs, a four-wavelength FBG with same sub FBG's spectrum was fabricated on a hydrogen loaded single mode fiber.
\end{abstract}

Keywords: Fiber Bragg grating (FBG), multi-wavelength FBG, phase mask, multiple exposures

Citation: Jingsheng LV, Xiaolei ZHANG, Haifeng QI, Jian Guo, Gangding PENG, and Chang WANG, "Experimental Research on Multi-Wavelength FBG Fabrication Based on Multiple Exposure,” Photonic Sensors, 2015, 5(3): 273-277.

\section{Introduction}

The fiber Bragg grating (FBG) is an important optical filter, in the study of more than 20 years, various FBGs and fabrication methods have been introduced which play a great role in the fiber lasers, optical fiber sensors, and communication technology $[1,2]$. In addition to some special structure FBGs just like long period FBG [3], blazed FBG [4], sampled FBG [5], and multimode FBG [6] having a spectrum with multiply reflection band, the spectra of ordinary FBGs are all the basic single wavelength. Although several FBGs series-connected on a single fiber can realize multi-wavelength distribution, but the structure is not convenient to use for the long size. It is found that, in experiments, the FBG will not be bleached when exposed again on the same section of the fiber, but form multi reflection band corresponding to the force tensing on the fiber. In this paper, a new method of fabrication of multi-wavelength FBG in a fiber is introduced using the grating wavelength tension control technology. A four-wavelength FBG with a consistent spectrum shape was fabricated by multi exposures with different tensions on the fiber.

\section{Experiment}

The scanning exposure method of FBG 
fabrication based on the phase mask is considered as most flexible and reliable in various methods. It is not only easy to control the basic parameters of the FBG such as the wavelength, reflectivity, and length, but also convenient for refractive index modulation to achieve apodization, being chirped and phase-shifted on the FBG [7]. The fabrication system of FBG is illustrated in Fig. 1. The FBG is fabricated by UV scanning espousing by moving UV source or the piece of fiber. The wavelength range is determined by the period of the phase mask, while the exact wavelength is determined by the force pulled on the fiber when exposing, and the bigger the force is, the shorter the wavelength is [8].

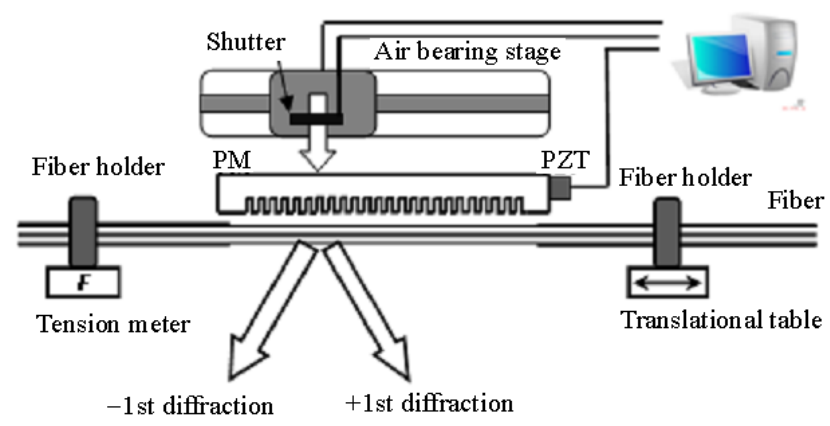

Fig. 1 Fabrication system of FBGs.

Hydrogen loading makes fiber high photosensitive, which ensures that expected results of refractive index modulation can be very well showed in the grating spectrum. The phase mask method has undemanding coherence of the ultraviolet (UV) light source, which allows us to use excimer lasing at $248 \mathrm{~nm}$. The FBG with a length of $10 \mathrm{~mm}$ was fabricated by setting the pulse energy of excimer laser as $10 \mathrm{~mJ}$ and the scanning velocity as $0.1 \mathrm{~mm} / \mathrm{s}$ during each exposure. Firstly, the FBG with a central wavelength of $1551.33 \mathrm{~nm}$ and reflectivity of $-31 \mathrm{~dB}$ was fabricated under exposure at a frequency of $120 \mathrm{~Hz}$, while the tension was maintained at $50 \mathrm{~g}$ by the use of fiber holder and strain gauge during exposure, as shown in Fig.2(a). Then, the tension imposed on fiber was adjusted to be $100 \mathrm{~g}$, and the second scanning exposure at the same frequency of $120 \mathrm{~Hz}$ brought dual central wavelength to the FBG, which had a central wavelength of $1550.64 \mathrm{~nm}$ and a reflectivity of $-30.6 \mathrm{~nm}$. Meantime, the stop-band of the first layer of the grating moved to $1551.54 \mathrm{~nm}$, and its reflectivity changed into $-36 \mathrm{~dB}$, with an increase in the central wavelength and reflectivity simultaneously as shown in Fig. 2(b).
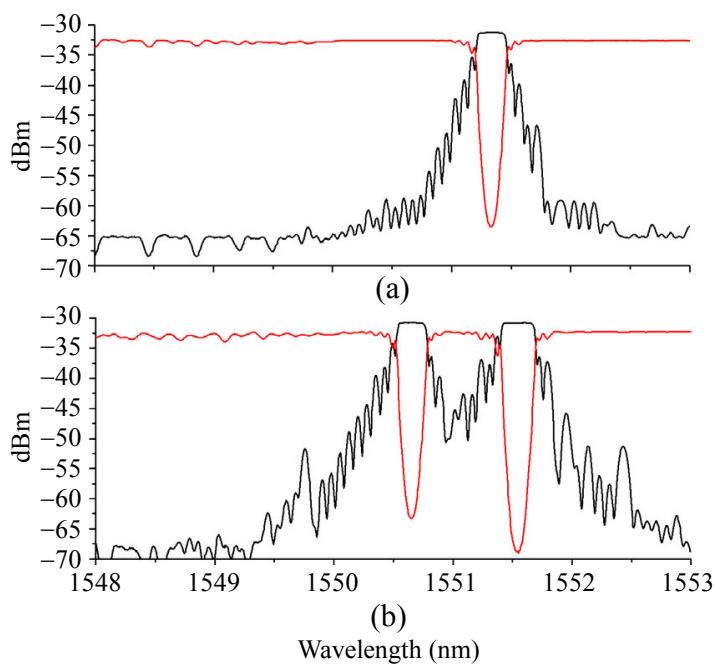

Fig. 2 Spectra change of the FBG: (a) first exposure at $50 \mathrm{~g}$ tensile force and (b) second exposure at $100 \mathrm{~g}$ tensile force.

If the positions of the two layers of the grating are staggered by $2 \mathrm{~mm}$, i.e. the total grating length is $12 \mathrm{~mm}$, and their overlapping part is $8 \mathrm{~mm}$, the spectra of the two gratings will both loss their symmetry as single-wavelength, short wave oscillation issue emerges, and the reflection is weaker than that of the single grating, as shown in Fig. 3.

Based on the above experimental results, the four-wavelength FBG was designed and fabricated after many calibrating tests. Each layer of the sub-grating was fabricated on hydrogen loaded sensitive enhanced single-mode fiber with the UV pulse energy of $10 \mathrm{~mJ}$ and scanning velocity of $0.1 \mathrm{~mm} / \mathrm{s}$, with the exposure frequency and fiber tension as shown in Table 1. The four-wavelength FBG had a uniform wavelength interval within $0.05 \mathrm{~nm}$ error and a reflection of the sub-grating of around $-23 \mathrm{~dB}$ with the good consistency 
as shown in Fig. 4.

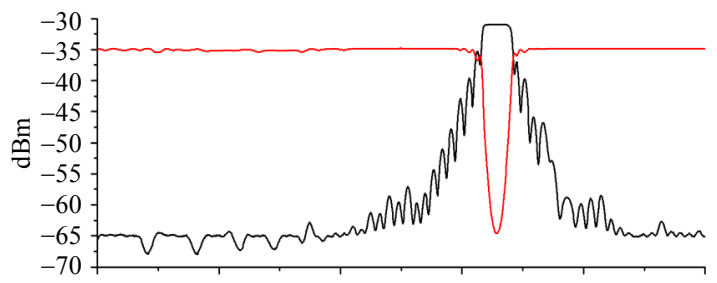

(a)

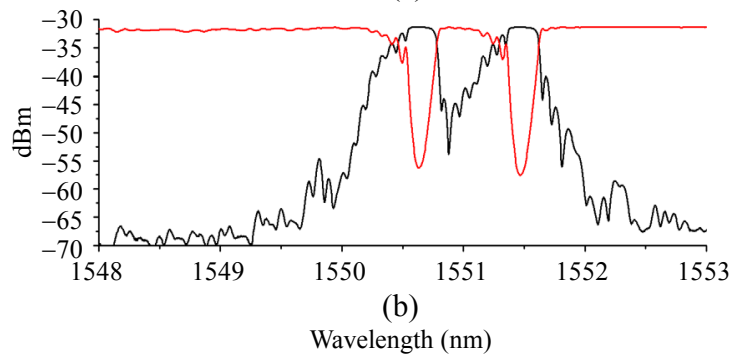

Fig. 3 Spectra of the double-wavelength FBG with $2 \mathrm{~mm}$ dislocation: (a) first exposure at $50 \mathrm{~g}$ tensile force and (b) second exposure at $100 \mathrm{~g}$ tensile force.

Table 1 Fabrication conditions of every sub-FBG.

\begin{tabular}{c|c|c|c|c}
\hline & 1 st level & 2nd level & 3rd level & 4th level \\
\hline Exposure frequency & $100 \mathrm{~Hz}$ & $110 \mathrm{~Hz}$ & $120 \mathrm{~Hz}$ & $130 \mathrm{~Hz}$ \\
\hline Tensile force & $50 \mathrm{~g}$ & $100 \mathrm{~g}$ & $150 \mathrm{~g}$ & $200 \mathrm{~g}$ \\
\hline
\end{tabular}

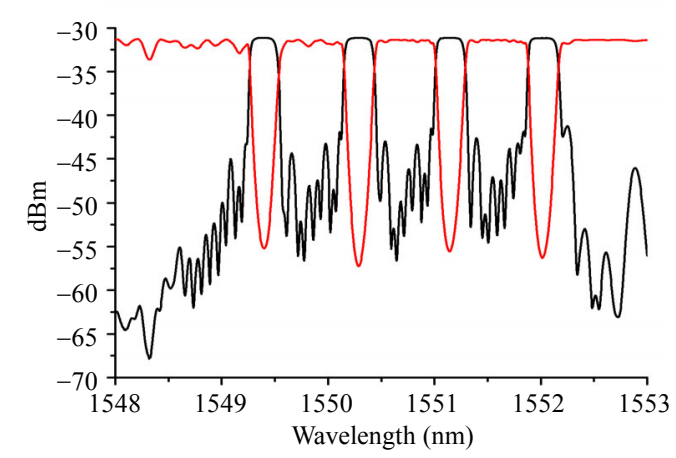

Fig. 4 Spectra of a four-wavelength FBG fabricated on $\mathrm{H}_{2}$-loading photosensitive fiber.

And Nufern GF1 germanium-doped photosensitive fiber was also used to repeat the fabrication as above. The results reveal that the multi-wavelength FBG can also be written on germanium-doped fiber. However, the reflectivity of all the sub-gratings decrease significantly with more layers of the grating no matter how we change the exposure, as shown in Fig. 5.

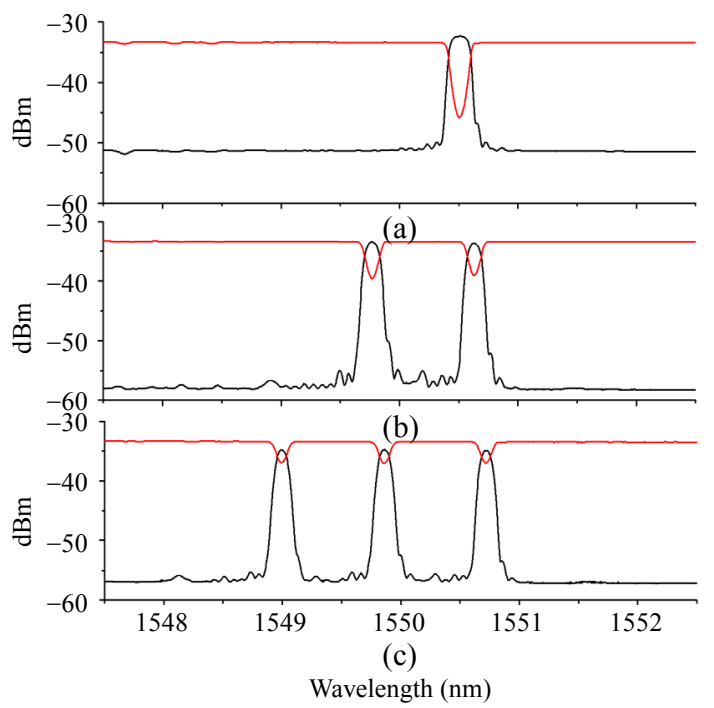

Fig. 5 Spectra of a three-wavelength FBG fabricated on Ge-doped photosensitive fiber.

\section{Discussion}

As we all known, the Bragg wavelength and reflection of the normal FBG can be described by $\lambda_{B}=2\left(n_{0}+\Delta n_{\text {eff }}\right) \Lambda_{g}$ and $R=\tanh ^{2}\left(\frac{\pi}{\lambda_{B}} v \Delta n L\right)[9$, 10], while $n_{0}$ is the refractive index of the core, $\Delta n_{\text {eff }}$ is an average increase induced by UV exposure, $\Delta n$ is the amplitude of refractive index modulation, $v$ is the fringe visibility of refractive index modulation, and $\Lambda_{g}$ is the grating period. If the larger tension is applied on the fiber during exposure, the smaller $\Lambda_{g}$ is obtained after the tension is released, therefore the shorter wavelength FBG is got.

The wavelength of sub-gratings formed first under multiple exposure increases with larger $\Delta n_{\text {eff }}$ under accumulation of local exposure no matter what kind of exposure technology or photosensitive fiber is used. Due to the lower coherence of excimer laser, the $v$ value of the FBG is so small that the high reflection depends on an increase in $\Delta n$ under strong exposure [11]. For hydrogen loaded photosensitive fiber, although the $v$ value of each layer of the grating is getting weaker during interaction, the relative change is finite, and $\Delta n$ of each layer of the grating 
increases during intercoupling. Hence, the reflection of the sub-grating increases. While $\Delta n$ of germanium doped photosensitive fiber is nearly one magnitude lower than that of hydrogen loaded photosensitive fiber under the same exposure, attributing to its limited photosensitivity [12], the weakening of $v$ is more obvious than an increase in $\Delta n$, which results in lower reflection.

The responses of the fabricated four-wavelength FBG to temperature and strain were tested individually as shown in Figs. 6 and 7, where perfectly consistent performances were obtained due to the same material property of the four sub-gratings. Despite no superiority to the single wavelength applied in fiber sensing based on the wavelength demodulation technology, this could be quite valuable for the fiber laser and fiber filter [13-15].

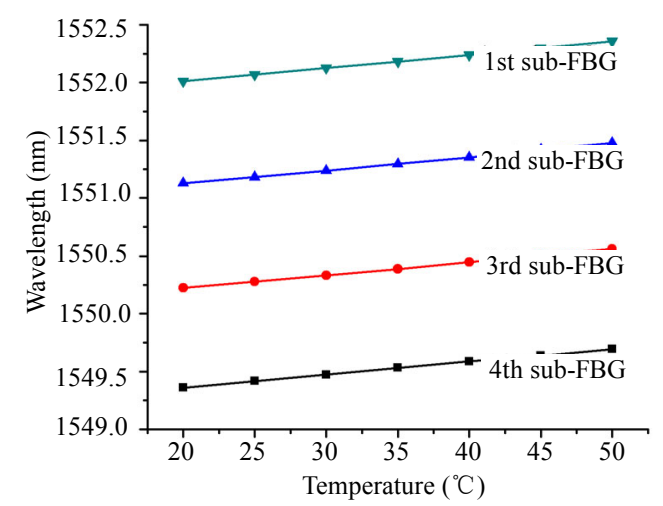

Fig. 6 Temperature response of the four-wavelength FBG.

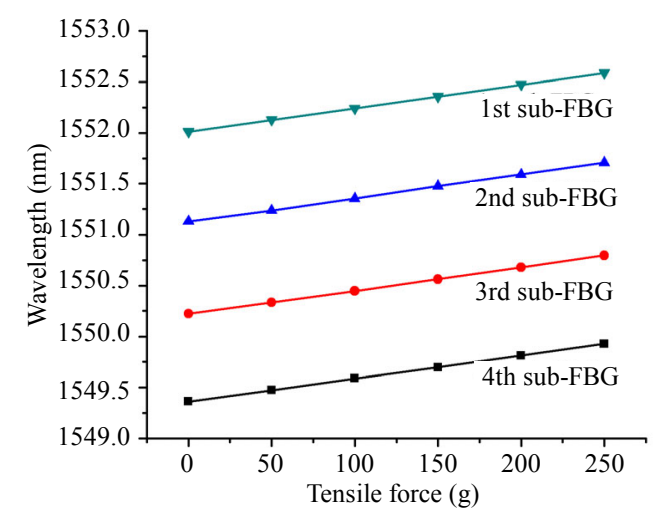

Fig. 7 Tension response of the four-wavelength FBG.

\section{Conclusions}

Based on the scanning exposure method by the use of the excimer laser and phase mask, a novel multiple exposure method was realized to fabricate the multiple-wavelength FBG at the same position. The multiple-wavelength FBG is easier to be fabricated on hydrogen loaded photosensitive fiber than on germanium doped photosensitive fiber. The FBG length and position should be kept consistent to keep the spectrum of the sub-grating from short wavelength oscillation. The sub-gratings of the multiple-wavelength FBG have the same wavelength to temperature and strain response. The multiple-wavelength FBGs with different wavelength intervals can be obtained by the FBG wavelength and tension control technology. It will pay a role in the fiber laser and fiber filter, etc.

\section{Acknowledgement}

The authors give thanks to the support by National Nature Science Foundation of China under Grant No. 61205083 and Shandong Provincial Nature Science Foundation under Grant No. ZR2012FQ002.

Open Access This article is distributed under the terms of the Creative Commons Attribution License which permits any use, distribution, and reproduction in any medium, provided the original author(s) and source are credited.

\section{References}

[1] J. Wang, T. Liu, G. Song, H. Xie, L. Li, X. Deng, et al., "Fiber Bragg grating (FBG) sensors used in coal mines," Photonics Sensors, 2014, 4(2): 120-124.

[2] S. Liaw, W. Wu, and C. Shin, "Tunable L-band fiber lasers using fiber bragg gratings and wavelength reflector," Microwave and Optical Technology Letters, 2014, 56(11): 2681-2683.

[3] C. Wang and G. Zhang, "Progress in fabrication method of long period fiber grating," Transducer and Microsystem Technologies, 2007, 26(1): 1-4.

[4] D. Yang, L. Du, Z. Xu, Y. Jiang, J. Xu, M. Wang, et al., "Magnetic field sensing based on tilted fiber Bragg grating coated with nanoparticle magnetic fluid," Applied Physics Letters, 2014, 104(6): 061903-1-061903-3.

[5] Y. Liu, L. Wang, P. Tao, S. Feng, G. Yin, W. Ren, et al., "Output characteristics of wavelength tunable 
fiber lasers based on sampled Bragg gratings," Acta Physica Sinica, 2011, 60(2): 024207-1-024207-7.

[6] H. Pan, X. Yang, Z. Tong, H. Gao, and F. Wei, "Dual-wavelength fiber laser based on er-doped fiber ring filter and multimode FBG," Infrared and Laser Engineering, 2012, 41(9): 2317-2320.

[7] Z. Song, H. Qi, G. Peng, S. Li, J. Guo, and C. Wang, "Research on fabrication technology of fiber Bragg grating by phase mask moving," Journal of Optoelectronics Laser, 2014, 25(7): 1294-1297.

[8] Z. Song, H. Qi, G. Peng, S. Li, J. Guo, and C. Wang, "Reaearch on control technology of fiber grating wavelength by pulling force in grating fabrication," ACTA Optica Sinica, 2013, 33(7): 0706009-1-0706009-5.

[9] E. Turan, "Fiber grating spectra," Journal of Lightwave Technology, 1997, 15(8): 1277-1294.

[10] K. O. Hill and G. Meltz. "Fiber Bragg grating technology fundamentals and overview," Journal of
Lightwave Technology, 1997, 15(8): 1263-1276.

[11] H. Jia and Y. Li, "Saturation effect of fiber Bragg gratings," ACTA Photonica Sinica, 2000, 29(9): 801-805.

[12] J. Li and D. Jiang, "Hydrogen loading and photolytic index changes in germanosilicate fiber," Journal of Inorganic Materials, 2006, 21(2): 345-350.

[13] Z. Tan, J. Gao, Y. Chen, Y. Liu, T. Ning, and S. Jian, "Multi-wavelength dispersion compensator based on fiber gratings with low crosstalk," ACTA Physica Sinica, 2007, 56(1): 274-279.

[14] J. Li, Z. Ran, Y. Ma, H. Liu, and S. Yan, "Dynamic strain sensing characteristics of superimposed fiber Bragg gratings," Journal of Optoelectronics-Laser, 2013, 24(9): 1667-1672.

[15] G. Sun, J. Zheng, R. Wang, T. Pu, Z. Wei, J. Xiong, et al., "Research on spectra of superimposed fiber Bragg grating," Journal of Optoelectronics-Laser, 2012, 23(12): 2249-2254. 